\title{
Effectiveness and safety of sofosbuvir/velpatasvir \pm ribavirin vs glecaprevir/pibrentasvir in genotype 3 hepatitis C virus infected patients
}

\author{
Luis Margusino-Framiñán, 1,2 Purificación Cid-Silva, 1,2 Sandra Rotea-Salvo, ${ }^{1}$ \\ Álvaro Mena-de-Cea, ${ }^{2,3}$ Francisco Suárez-López, ${ }^{4}$ Pilar Vázquez-Rodríguez, ${ }_{1}^{2,3}$ \\ Manuel Delgado-Blanco, ${ }^{2,4}$ Ana Isabel Sanclaudio-Luhia, ${ }^{5}$ Isabel Martín-Herranz, ${ }^{1}$ \\ Ángeles Castro-Iglesias, 2,3
}

\begin{abstract}
${ }^{1}$ Pharmacy Service, Universitary Hospital of A Coruña, A

Coruña, Spain

${ }^{2}$ Division of Clinical Virology,

Biomedical Research Institute of A Coruña (INIBIC),

Universitary Hospital of A Coruña (CHUAC), Sergas, University of A Coruña (UDC), A Coruña, Spain

${ }^{3}$ Infectious Diseases Unit. Internal Medicine Service, Universitary Hospital of A Coruña (CHUAC), A Coruña, Spain

${ }^{4}$ Hepatology Unit, Digestive System Service, University Hospital of A Coruña (CHUAC) A Coruña, Spain

Information Systems Department, University Hospital of A Coruña, A Coruña, Spain
\end{abstract}

\section{Correspondence to}

Dr Luis Margusino-Framiñán, Pharmacy, XXI A Coruña, A Coruña 15006, Spain; Luis. Margusino.Framinan@sergas.

Received 6 August 2019 Revised 14 January 2020 Accepted 16 January 2020 Published Online First 7 February 2020

EAHP Statement 6: Education and Research.

\section{Check for updates}

(C) European Association of Hospital Pharmacists 2020. No commercial re-use. See rights and permissions. Published by BMJ.

To cite: MargusinoFramiñán L, Cid-Silva P, Rotea-Salvo $\mathrm{S}$, et al. Eur I Hosp Pharm 2020:27:e41-e47.

\section{ABSTRACT}

Objectives Sofosbuvir/velpatasvir \pm ribavirin (SOF/ VEL \pm RBV) and glecaprevir/pibrentasvir (GLE/PIB) are the drug combinations of choice for treating individuals with genotype 3 hepatitis C virus (G3-HCV) infection. The objective of this study was to evaluate the effectiveness and safety of SOF/VEL \pm RBV compared with GLE/PIB for treating G3-HCV infection under routine clinical practice conditions.

Methods We conducted a prospective observational cohort study of individuals with G3-HCV infection who initiated treatment with SOF/VEL +/-RBV or GLE/ PIB between April 2017 and July 2018. Prisoners and children were excluded. The outcome variable of effectiveness was sustained virological response 12 weeks after completing treatment (SVR12). The safety variable was withdrawal secondary to severe adverse events (SAEs). Covariates included sex, age, HIV coinfection, previous liver transplant, cirrhosis, hepatic fibrosis and previous antiviral treatment. Statistical significance was calculated using Fisher's exact test or the Mann-Whitney U-test.

Results A total of 76 patients were included in the analysis, of whom 46 were treated with SOF/ $V E L \pm R B V$ and 30 were treated with GLE/PIB. No baseline differences were observed between treatment groups with respect to age, sex, HIV co-infection, fibrosis stage, cirrhosis and previous antiviral treatment. Of the patients treated with SOF/VEL \pm RBV and GLE/PIB, 95.7\% and $96.7 \%$ reached SVR12, respectively $(P=0.7)$. Of patients with and without cirrhosis, $83.3 \%$ and $98.4 \%$ reached SVR12, respectively $(P=0.09)$. Of the patients with low-grade hepatic fibrosis (FO-2) and advanced fibrosis (F3-4), 100\% and $85.7 \%$ reached SVR12, respectively $(\mathrm{P}=0.03)$. In treatment-naïve and treatment-experienced patients, $95.7 \%$ and $100 \%$ reached SVR 12 , respectively $(P=0.57)$, without significant differences independent of the treatment group ( $P=0.28$ for SOF/VEL $\pm R B V ; P=0.18$ for GLE/PIB). The incidence of AEs was $21.1 \%(95 \% \mathrm{CI}$ $11.3 \%$ to $30.9 \%$ ). None of the patients developed an SAE or required antiviral treatment withdrawal.

Conclusions SOF/VEL \pm RBV or GLE/PIB are safe and effective for treating G3-HCV-infections, with a lower effectiveness in patients with advanced fibrosis F3-4.

\section{INTRODUCTION}

It is estimated that the prevalence of chronic hepatitis $\mathrm{C}$ virus (HCV) infection in Europe is approximately
$1.1 \%$, with a total affected population of 5.6 million people. ${ }^{1}$ The prevalence of HCV genotypes varies among regions, with genotype 3 (G3) being the second most prevalent in Europe after genotype 1 (G1), accounting for approximately $25 \%$ of cases of chronic hepatitis $\mathrm{C}(\mathrm{CHC})^{2}$ Compared with G1-HCV, G3-HCV chronic infection has a faster progression to liver cirrhosis $^{3-6}$ and hepatocellular carcinoma (HCC), ${ }^{7}$ and is associated with a higher incidence of hepatic steatosis. ${ }^{8-11}$ Furthermore, compared with other genotypes, G3-HCV has been reported to exhibit lower rates of sustained virologic response (SVR) with direct-acting antivirals (DAAs), particularly in patients with advanced liver fibrosis and non-responders to previous treatment. ${ }^{12}$ Therefore, the evaluation of the effectiveness of antiviral treatment against G3-HCV chronic infection under clinical practice conditions in the era of DAAs is of special interest.

The treatment of G3-HCV recommended by scientific societies has changed in recent years. Taking the results of clinical studies into account, the European Association for the Study of the Liver (EASL) has repeatedly updated its recommendations, which have advised the use of peginterferon (PegIFN) + ribavirin (RBV) (2011), sofosbubir (SOF) + PegIFN+RBV, SOF+RBV (2013, 2015), SOF+daclatasvir $(\mathrm{DAC}) \pm \mathrm{RBV}, \mathrm{SOF} / \mathrm{velpatasvir}(\mathrm{VEL}) \pm \mathrm{RBV}$ (2016), SOF/VEL, glecaprevir/pibrentasvir (GLE/PIB) and SOF/VEL/voxilaprevir (VOX) (2018). ${ }^{13-17}$ The Infectious Diseases Society of America (IDSA) and American Association for the Study of the Liver (AASLD) have also updated their treatment recommendations, with the most recent update being in $2018 .^{18}$ Table 1 provides a summary of the current treatment recommendations by EASL and IDSA/AASLD. ${ }^{17} 18$ However, there have been no studies published that compare the safety and effectiveness of these therapies in individuals with G3-HCV infection.

The objective of this study was to evaluate the effectiveness and safety of 8- to 24-week treatment regimens of SOF/VEL \pm RBV and GLE/PIB for treating G3-HCV infection under routine clinical practice conditions.

\section{METHODS}

\section{Study design and patient selection}

We conducted an observational, prospective, cohort study of patients with G3-HCV infection who started HCV treatment with SOF/VEL \pm RBV or GLE/PIB 
Table 1 Adaptation of the treatment recommendations of EASL and IDSA/AASLD of patients infected with genotype 3 of the hepatitis C virus, without cirrhosis or with compensated cirrhosis. recommended treatments.Update $2018 * \dagger^{1718}$

\begin{tabular}{|c|c|c|}
\hline Genotype 3-infected patient & EASL $^{17}$ & IDSA/AASLD ${ }^{18}$ \\
\hline Naïve-non cirrhotic & $\begin{array}{l}\text { SOF/VEL } 12 w \\
\text { GLE/PIB } 8 w\end{array}$ & $\begin{array}{l}\text { SOF/VEL } 12 w \\
\text { GLE/PIB } 8 w\end{array}$ \\
\hline $\begin{array}{l}\text { Treatment experienced-non } \\
\text { cirrhotic } \neq\end{array}$ & $\begin{array}{l}\text { SOF/VEL } 12 w \\
\text { GLE/PIB } 12 w\end{array}$ & SOF/VEL \pm RBV $12 \mathrm{w}$ \\
\hline Naïve-cirrhotic & $\begin{array}{l}\text { GLE/PIB } 12 \text { w } \\
\text { SOF/VEL/VOX } 12 \text { w }\end{array}$ & $\begin{array}{l}\text { SOF/VEL } 12 w \\
\text { GLE/PIB } 12 w\end{array}$ \\
\hline Treatment experienced-cirrhotic & $\begin{array}{l}\text { SOF/VEL/VOX } 12 \mathrm{w} \\
\text { GLE/PIB } 16 \mathrm{w}\end{array}$ & $\begin{array}{l}\text { ELB/GRZ+SOF } 12 w \\
\text { SOF/VEL/VOX } 12 w\end{array}$ \\
\hline
\end{tabular}

${ }^{*}$ This table is a simplified adaptation. It is recommended to consult the direct references.

†Ribavirin addition is recommended in some subgroups of patients. ¥Recommendations in experienced patients are different and vary depending on whether the previous treatment was based on peginterferon or DAAs. AASLD, American Association for the Study of the Liver; EASL, European Association for the Study of the Liver; ELB, elbasvir; GLE, glecaprevir; GRZ, grazoprevir; IDSA, Infectious Diseases Society of America; $\mathrm{PIB}$, pibrentasvir; RBV, ribavirina; SOF, sofosbuvir; VEL, velpatasvir; VOX, voxilaprevir.

between April 2017 and July 2018 and had reached week 12 post-treatment by January 2019. Infectious disease specialists and hepatologists chose the antiviral treatment regimen, taking into account not only the prevailing clinical practice conditions and international recommendations, ${ }^{17}{ }^{18}$ but also variables such as concomitant treatment, lifestyle habits or patient preferences. The therapeutic regimen was a daily fixed combination of SOF $400 \mathrm{mg} / \mathrm{VEL} 100 \mathrm{mg}$ (Epclusa; Gilead Sciences International Ltd) with or without the addition of RBV (Ribavirina Normon; Normon Laboratory), adjusted according to body weight and patient characteristics for 12 to 24 weeks, or three GLE $100 \mathrm{mg} /$ PIB $40 \mathrm{mg}$ fixed-dose combination tablets (Maviret; Abbvie Spain) administered for $8-16$ weeks. The length of treatment was adjusted to the therapeutic guidelines, taking the patient characteristics, including the presence of cirrhosis, previous antiviral treatment and hepatic decompensation, into account. ${ }^{17} 18$ The inclusion criteria selected adult patients ( $\geq 18$ years of age) with G3-HCV chronic infection. The patients included in the analysis were permitted to be naïve or treatment-experienced to peg-INF+RBV or DAAs, in all stages of hepatic fibrosis, including patients with decompensated cirrhosis or portal hypertension. Patients with HIV co-infection and those who had had a previous liver transplant were included.

\section{Effectiveness and safety variables}

Antiviral effectiveness and safety evaluation were carried out through SiMON, ${ }^{19}$ a local intelligent artificial monitoring system designed specifically for $\mathrm{CHC}$ patients. This system records data from the clinical history, related virological response and adverse events (AE). Additional data regarding hospitalisations or admissions to the emergency room were collected from patients' electronic clinical records.

HCV viral load was measured using the real-time PCR technique, with the Cobas AmpliPrep platform (Roche Molecular Systems, Basel, Switzerland) and the HCV Quantitative Test, version 2.0. The limits of detection and quantification in plasma were $11 \mathrm{IU} / \mathrm{mL}$ (95\% CI): $10-13 \mathrm{IU} / \mathrm{mL}$ ) for the lower limit of detection (LOD), with a $95 \%$ positivity rate and $15 \mathrm{UI} / \mathrm{mL}$ as the LOD with positive results. Viral load was measured at baseline, on completion of treatment and 12 weeks after completion of treatment. Transient elastography was used for the staging of liver fibrosis (Fibroscan, Echosens, Paris, France), and patients were stratified according to stiffness results into fibrosis $\mathrm{F} 0-1$ $(<7.6 \mathrm{kPa})$, F2 $(7.6-9.5 \mathrm{kPa}), \mathrm{F} 3(9.6-14.4 \mathrm{kPa})$ or F4 $(>14.4 \mathrm{kPa}$ in HCV mono-infected patients and $>14.0 \mathrm{kPa}$ in HIV co-infected patients)..$^{21}$

Adherence rates were calculated following continuous measurement of the medication acquisition (CMA) method, ${ }^{22}$ during the monthly visits to the Hospital Pharmacy Service where the study was conducted. This method measured cumulative days' supply obtained over a series of intervals/total days from the beginning to the end of the time period. Drug-drug interactions (DDIs) were identified by the clinical team (clinical pharmacists, hepatologists and infectious disease specialists) using the Hep Drug Interactions database of the University of Liverpool, ${ }^{23}$ recommended as reference by EASL. ${ }^{17}$ If there was no information available in this database, Lexicomp Drug Interactions, ${ }^{24}$ IBM Micromedex,${ }^{25}$ analysis of pharmacokinetic parameters available in the technical data sheet and consultation with the DAA manufacturing laboratory were employed.

The primary effectiveness endpoint was the proportion of patients with SVR12, defined as an undetectable HCV ribonucleic acid (HCV-RNA) 12 weeks' post-treatment. Secondary efficacy variables included treatment failure (detectable HCV-RNA in a patient with previous undetectable HCV-RNA on treatment), relapse (detectable HCV-RNA 12 weeks' post-treatment in a patient with undetectable HCV-RNA at the end of treatment), virological failure (HCV-RNA level remaining above the LOD throughout treatment) or missing HCV-RNA data 12 weeks' post-treatment due to on-treatment withdrawal secondary to severe AEs (SAEs) or death.

The primary safety endpoint was the percentage of treatment withdrawal secondary to SAEs. Secondary variables included the patients' self-referred AEs (stratified into mild, moderate or severe), emergency room consultation and hospital admissions secondary to SAEs.

\section{Statistical analysis}

Data of baseline variables, primary or secondary effectiveness variables, and safety end-points were collected and analysed by an intention-to-treat analysis according to the treatment regimen. Quantitative variables were expressed as the mean $\pm S D$ deviation (SD) and were analysed using the Student's $t$-test or the Mann-Whitney $U$-test according to data distribution. Qualitative variables were expressed as counts and percentages, and were compared using a Chi-square test or Fisher's exact test. Primary end-points were expressed as a percentage and exact 95\% binomial CI. To determine the influence of baseline factors on the primary end-points, relative risk with a 95\% CI (Katz) for cohort studies was calculated using the Chi-square association test without Yates correction or Fisher's exact bilateral test, according to the number of outcome events. To detect differences between treatment subgroups and predictors of response, univariate and multivariate analyses were performed. The results were considered to be statistically significant when the P-value was $<0.05$. Statistical analysis was carried out using the Epidat 4.2 software.

\section{Ethical aspects}

This study complied with the Declaration of Helsinki of Good Clinical Practices. It was classified as 'Observational PostAuthorisation Study with Human Medicines' by the Spanish Agency of Medicines and Medical Devices (AEMPS) (LMFNAA-2019-01), and was approved by the Clinical Research 


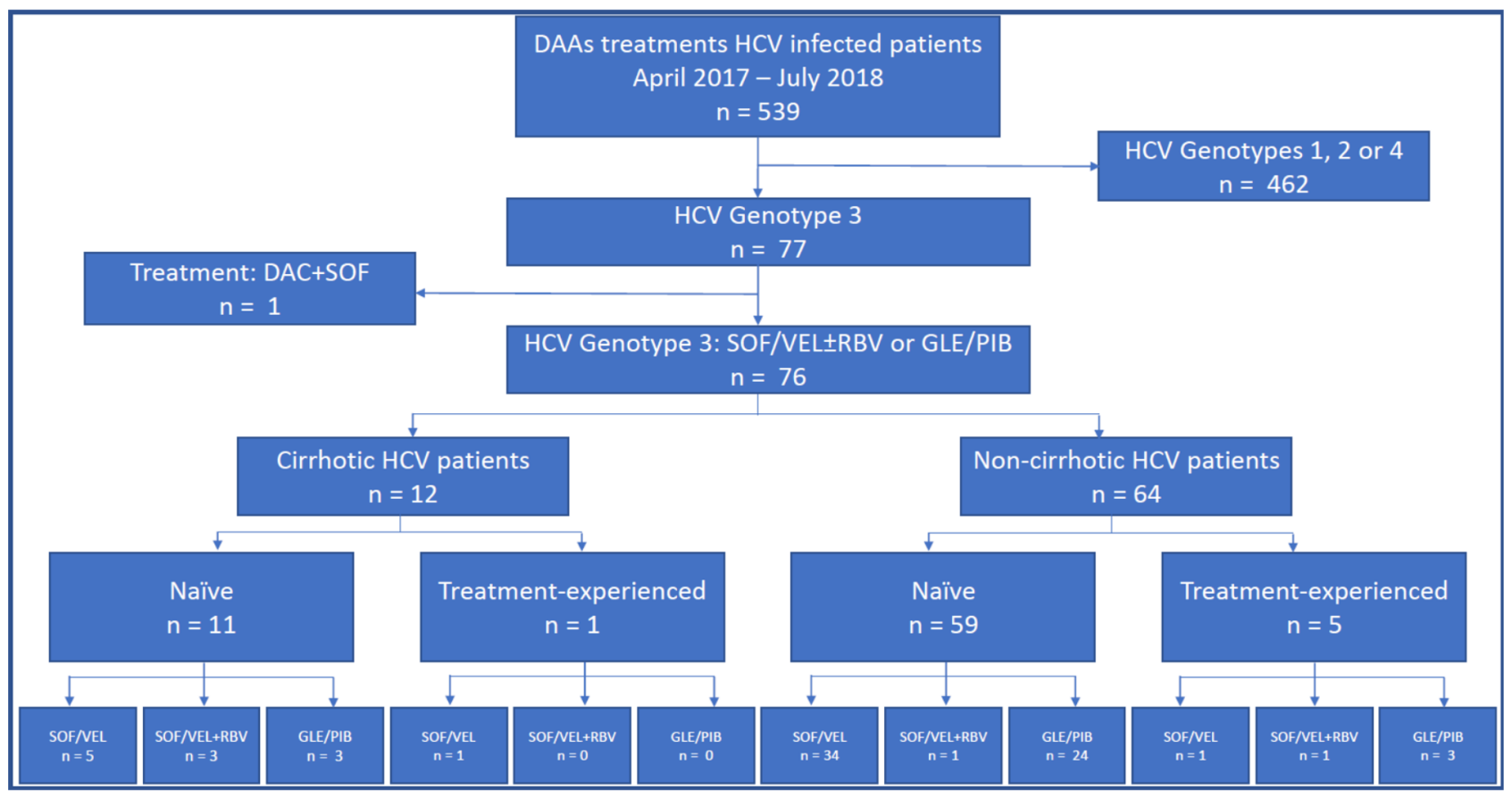

Figure 1 Study flow design.DAAs: direct-acting antivirals; HCV: hepatitis C virus; DAC: daclatasvir; SOF: sofosbuvir; VEL, velpatasvir; RBV: ribavirin; GLE: glecaprevir; PIB: pibrentasvir.

Ethics Committee (CREC) of the Regional Health Service (number 2016/161). Patients provided written informed consent and all study data were anonymised.

\section{RESULTS}

\section{Baseline patient demographics and characteristics}

A total of 539 adult patients started antiviral treatment during the study period at our institution, of which $76(14 \%)$ were infected with G3-HCV, and were treated with SOF/VEL \pm RBV or GLE/ PIB. Figure 1 shows the study flow design. Forty-six patients were treated with SOF/VEL \pm RBV for 12 weeks and 30 with GLE/PIB for $8-16$ weeks. The average adherence to SOF/VEL \pm RBV and GLE/PIB were $99.5 \pm 1.54$ vs $99.9 \pm 0.67$, respectively $(\mathrm{P}=0.13)$. Between concomitant treatments, $26 \%$ of patients in the SOF/ VEL \pm RBV group, and $3 \%$ of patients in the GLE/PIB group, presented with DDIs $(\mathrm{P}=0.02)$. With both drug regimens, there was a potential for DDIs with omeprazole. DDIs were managed according to reference recommendations, ${ }^{23}$ either by temporary suspension of omeprazole or by SOF/VEL administration with food: they were taken 4 hours before omeprazole at a maximum dose of $20 \mathrm{mg}$. The majority of patients were men (74\%) with a median age of 65 years, naïve to antiviral treatment, with HCV mono-infection and without advanced fibrosis (74\% F0-2). Only one patient $(3 \%)$ suffered hepatic decompensation before the start of antiviral treatment. Only one patient had a liver transplant. Of the patients, $98 \%$ of those treated with SOF/VEL, and $100 \%$ of those treated with SOF/VEL+RBV completed 12 weeks of treatment, and $80 \%$ of those treated with GLE/PIB completed 8 weeks of treatment. Table 2 shows the main baseline patient characteristics of both cohorts.

\section{Effectiveness outcomes}

All patients achieved virologic suppression at the end of treatment, but three of them relapsed within 12 weeks of follow-up, so the overall SVR12 was $96 \%$. The SVR12 was $96 \%$ in patients treated with SOF/VEL \pm RBV compared with $97 \%$ in patients treated with GLE/PIB $(\mathrm{P}=0.7)$ (table 3 ). The SVR12 was $83 \%$ in cirrhosis patients, compared with $98 \%$ in patients without cirrhosis $(\mathrm{P}=0.09)$. The SVR12 was $100 \%$ in patients with low fibrosis, compared with $86 \%$ in those with high fibrosis $(\mathrm{P}=0.03)$. The SVR12 was $96 \%$ in all treatment-naïve patients, compared with $100 \%$ in treatment-experienced patients $(\mathrm{P}=0.57)$. Both of the patients who experienced viral rebound were treated with SOF/VEL+RBV and completed 12 weeks of treatment, but both had severe fibrosis (one F3, and one F4). The patient who showed treatment failure with GLE/PIB was treated for 12 weeks but also had severe fibrosis (F4). Figure 2 shows the SVR12 according to the basal fibrosis stage. The cirrhosis patient with hepatic decompensation, and the patient who had had a liver transplant both achieved SVR12. The SVR12 was 83\% in the six patients with HIV co-infection, compared with $97 \%$ in patients with mono-infection $(\mathrm{P}=0.57)$. No other baseline patient or treatment factors that could influence treatment effectiveness were identified, and there were no significant differences in SVR12 according to the patient's sex, basal HCV viral load, platelet count or serum albumin concentration.

Multivariate analysis of the influence of the presence or absence of cirrhosis, previous hepatic decompensation, previous antiviral treatments based on PegIFN and current antiviral treatment (SOF/VEL \pm RBV or GLE/PIB) did not identify any variables that were independently associated with SVR12.

\section{Safety outcomes}

During follow-up, 21\% of patients experienced an AE, and 9\% of patients experienced a moderate $\mathrm{AE}$. The incidence of any degree $\mathrm{AE}$ was $26 \%$ in patients who received SOF/VEL \pm RBV vs $13 \%$ in patients who received GLE/PIB $(\mathrm{P}=0.30)$. Moderate AEs secondary to SOF/VEL \pm RBV included fatigue/asthenia, ocular pain, anxiety, dry skin, irritability and insomnia. Moderate AEs secondary to GLE/PIB included only fatigue/asthenia. The incidence of moderate AEs was higher in patients with cirrhosis $(33 \%)$ compared with those without cirrhosis $(5 \%, \mathrm{P}=0.009)$. 
Table 2 Demographic and virological characteristics of the study population

\begin{tabular}{|c|c|c|c|}
\hline Characteristic & $\begin{array}{l}\text { Patients with } \\
\text { SOF/VEL } \pm \text { RBV }\end{array}$ & $\begin{array}{l}\text { Patients with } \\
\text { GLE/PIB }\end{array}$ & P-value \\
\hline Population, \% (n) & $60.5 \%(46)$ & $39.5 \%(30)$ & \\
\hline Males, \% (n) & $78.3 \%(36)$ & $66.7 \%(20)$ & 0.39 \\
\hline Age, mean (years $\pm S D$ ) & $48,72 \pm 9,41$ & $47,03 \pm 11,32$ & 0.5 \\
\hline Age $\geq 65$ years, \% (n) & $4.3 \%(2)$ & $6.6 \%(2)$ & 0.93 \\
\hline HIV co-infection, \% (n) & $13.0 \%(6)$ & $0.0 \%(0)$ & 0.1 \\
\hline \multicolumn{4}{|l|}{ Fibrosis stage, \% (n) } \\
\hline F0-1 & $54.3 \%(25)$ & $53.3 \%(16)$ & 0.88 \\
\hline F2 & $19.6 \%(9)$ & $16.7 \%(5)$ & 0.99 \\
\hline F3 & $6.5 \%(3)$ & $20.0 \%(6)$ & 0.16 \\
\hline F4 & $19.6 \%(9)$ & $10.0 \%(3)$ & 0.43 \\
\hline Previous clinical decompensation, \% (n) & $2.2 \%(1)$ & $0.0 \%(0)$ & 0.83 \\
\hline \multicolumn{4}{|l|}{ CTP classification, $\%$ (n) } \\
\hline A & $19.6 \%(9)$ & $10.0 \%(3)$ & 0.43 \\
\hline B & $0.0 \%(0)$ & $0.0 \%(0)$ & $>0.99$ \\
\hline C & $0.0 \%(0)$ & $0.0 \%(0)$ & $>0.99$ \\
\hline Hepatocellular carcinoma, \% (n) & $4.3 \%(2)$ & $0.0 \%(0)$ & 0.67 \\
\hline Liver transplant, \% (n) & $2.2 \%(1)$ & $0.0 \%(0)$ & 0.83 \\
\hline HCV viral load, log UI/mL (mean \pm SD) & $6,13 \pm 0.80$ & $5,97 \pm 0.95$ & 0.45 \\
\hline $\begin{array}{l}\text { Estimated glomerular filtration } \\
\text { rate }<60 \mathrm{~mL} / \mathrm{min}, \%(\mathrm{n})\end{array}$ & $2.2 \%(1)$ & $0.0 \%(0)$ & 0.83 \\
\hline \multicolumn{4}{|l|}{ Previous antiviral treatment, \% (n) } \\
\hline Naïve & $93,5 \%(43)$ & $90,0 \%(27)$ & 0.91 \\
\hline Treatment-experienced & $6,5 \%(3)$ & $10 \%(3)$ & \\
\hline \multicolumn{4}{|l|}{$\begin{array}{l}\text { Response to previous antiviral treatment, } \\
\% \text { (n) }\end{array}$} \\
\hline Recidivant & $0.0 \%(0)$ & $6.7 \%(2)$ & 0.3 \\
\hline Reinfection & $2.2 \%(1)$ & $0.0 \%(0)$ & 0.83 \\
\hline Null responder & $2.2 \%(1)$ & $0.0 \%(0)$ & 0.83 \\
\hline Unknown & $2.2 \%(1)$ & $3.3 \%(1)$ & 0.67 \\
\hline
\end{tabular}

CPT, Child-Pugh-Turcotte; GLE/PIB, glecaprevir/pibrentasvir; HCV, hepatitis C virus; HIV

human immunodeficiency virus; SOF/VEL $\pm R B V$, sofosbuvir/velpatasvir \pm ribavirin.
The incidence of AEs was not associated with sex, age or having previously been treated. Table 3 shows the main safety data. No patients experienced an SAE or required withdrawal, attention in the Emergency Department or hospitalisation as a result of an $\mathrm{AE}$, and none of the patients died during the study period.

\section{DISCUSSION}

DAAs currently recommended by international reference guidelines for individuals with G3-HCV infection include the use of four options of combinations of second-generation direct-acting antivirals, SOF/VEL \pm RBV, GLE/PIB, SOF/VEL/VOX or GZR/ $\mathrm{EBR}+\mathrm{SOF}$, depending on the baseline characteristics of the patient. ${ }^{17} 18$ These recommendations are based on the results of pivotal phase III clinical trials, where SVR12 rates obtained by any of these antiviral combinations are around 90\%-100\%, depending on the presence of cirrhosis and previous treatment (namely, variables that determine the antiviral selection, treatment length and/or RBV addition). ${ }^{26-32}$ Other variables that have be shown to influence the response to treatment, although not clinically significant, include the patient's sex, initial HCV viral load, serum albumin, platelet level and HIV co-infection. ${ }^{33} 34$ Our study has compared the effectiveness and safety of SOF/ $\mathrm{VEL} \pm \mathrm{RBV}$ vs GLE/PIB in routine clinical practice, and assessed the baseline and demographic characteristics of the patients in each treatment group to identify potential selection biases. No clinical differences between the treatment groups were observed at baseline in the proportion of treatment-naïve patients or patients with cirrhosis, so the two treatment groups were comparable. Likewise, both cohorts were balanced in relation to the patients' sex, age, liver elastography, baseline viral load and response to previous treatment among patients who had experienced previous treatment.

Based on the results of our real clinical practice study, SOF/ VEL \pm RBV or GLE/PIB show a high antiviral effectiveness in individuals infected with G3-HCV, with an overall SVR12 rate of $96 \%$ and no significant differences in the effectiveness

Table 3 Effectiveness and safety according with DAA

\begin{tabular}{|c|c|c|c|c|}
\hline Characteristic & Total $n=76 \%$ (n; Cl95\%) & Patients SOF/VEL $\pm R B V n=46$ & Patients GLE/PIB $n=30$ & P-value \\
\hline \multicolumn{5}{|l|}{ Virologic response } \\
\hline SVR12 (main) & $96,1(73 ; 88.9$ to 99.2$)$ & $95.7 \%(44)$ & $96,7 \%(29)$ & 0.7 \\
\hline Relapse & $3,9(3 ; 0.82$ to 11.1$)$ & $4,3 \%(2)$ & $3,3 \%(1)$ & \\
\hline Treatment failure & $0.0(0)$ & $0.0 \%(0)$ & $0.0 \%(0)$ & $>0.99$ \\
\hline Virologic failure & $0.0(0)$ & $0.0 \%(0)$ & $0.0 \%(0)$ & $>0.99$ \\
\hline Missing date & $0.0(0)$ & $0.0 \%(0)$ & $0.0 \%(0)$ & $>0.99$ \\
\hline Any grade drug-related AE & $21.1(16 ; 11.3$ to 30.9$)$ & $26,1 \%(12)$ & $13,3 \%(4)$ & 0.3 \\
\hline Moderate drug-related $\mathrm{AE}$ & $9.2(7 ; 2.1$ to 16.4$)$ & $8,7 \%(4)$ & $10.0 \%(3)$ & 0.83 \\
\hline \multicolumn{5}{|c|}{ Any grade AE with global incidence $>1.0 \%$ : } \\
\hline Fatigue/asthenia & $15.8(12 ; 6.9$ to 24.7$)$ & $17,4 \%(8)$ & $13,3 \%(4)$ & 0.88 \\
\hline Insomnia & $2.6(2 ; 0.3$ to 9.2$)$ & $2,2 \%(1)$ & $3,3 \%(1)$ & 0.67 \\
\hline Gastrointestinal upset & $1.3(1 ; 0.0$ to 7.1$)$ & $0,0 \%(0)$ & $2,2 \%(1)$ & 0.83 \\
\hline Headache & $1.3(1 ; 0.0$ to 7.1$)$ & $2,2 \%(1)$ & $0.0 \%(0)$ & 0.83 \\
\hline Anxiety & $1.3(1 ; 0.0$ to 7.1$)$ & $2,2 \%(1)$ & $0.0 \%(0)$ & 0.83 \\
\hline Diarrhoea & $1.3(1 ; 0.0$ to 7.1$)$ & $2,2 \%(1)$ & $0.0 \%(0)$ & 0.83 \\
\hline Irritability & $1.3(1 ; 0.0$ to 7.1$)$ & $2,2 \%(1)$ & $0.0 \%(0)$ & 0.83 \\
\hline Constipation & $1.3(1 ; 0.0$ to 7.1$)$ & $2,2 \%(1)$ & $0.0 \%(0)$ & 0.83 \\
\hline Ocular pain & $1.3(1 ; 0.0$ to 7.1$)$ & $2,2 \%(1)$ & $0.0 \%(0)$ & 0.83 \\
\hline Blurry vision & $1.3(1 ; 0.0$ to 7.1$)$ & $2,2 \%(1)$ & $0.0 \%(0)$ & 0.83 \\
\hline Dry skin & $1.3(1 ; 0.0$ to 7.1$)$ & $2,2 \%(1)$ & $0.0 \%(0)$ & 0.83 \\
\hline Dizziness & $1.3(1 ; 0.0$ to 7.1$)$ & $2,2 \%(1)$ & $0.0 \%(0)$ & 0.83 \\
\hline
\end{tabular}

AE, adverse event; DAA, direct-acting antiviral; GLE/PIB, glecaprevir/pibrentasvir; SOF/VEL \pm RBV, sofosbuvir/velpatasvir \pm ribavirin; SVR12, sustained virologic response week 12 . 


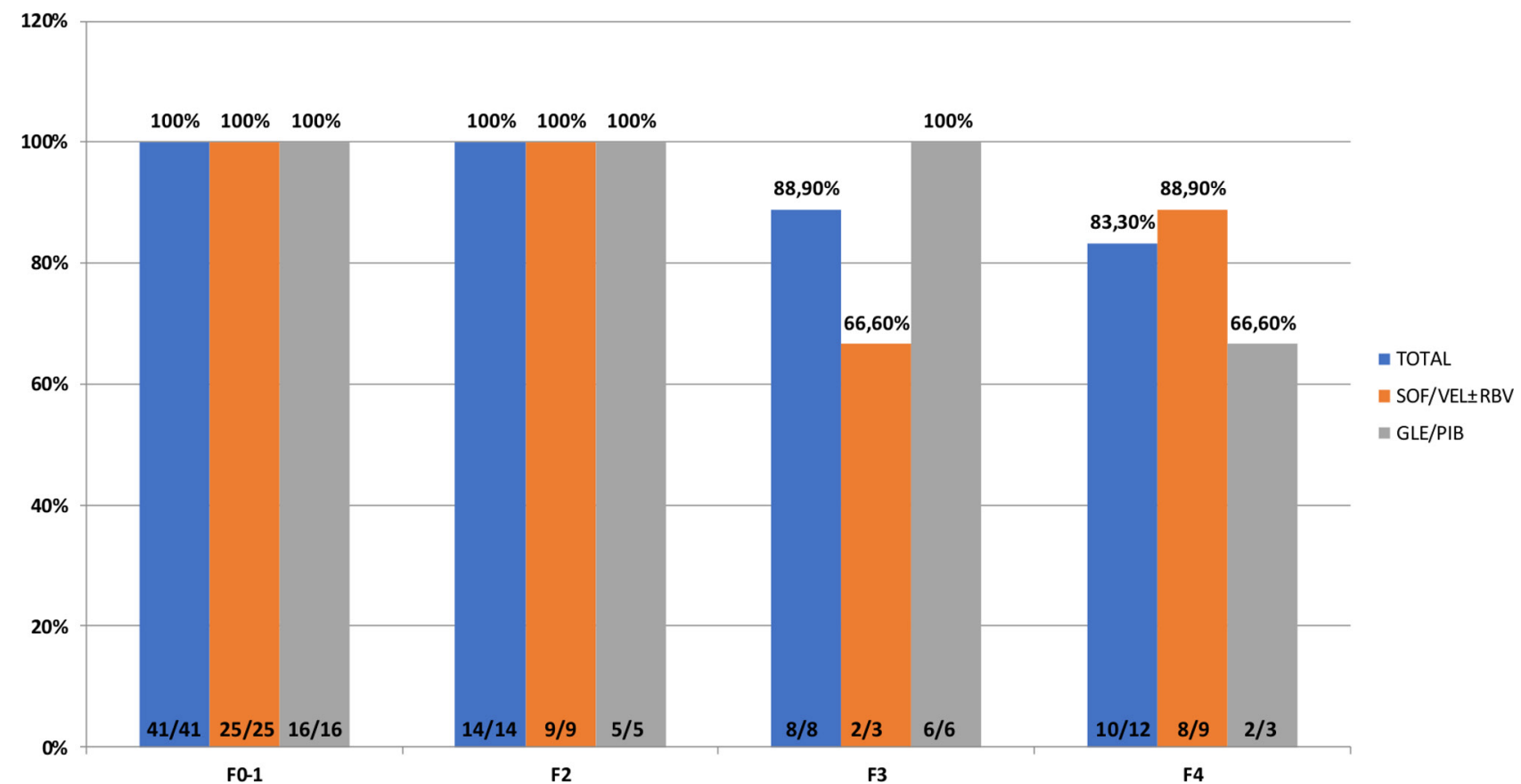

Figure 2 SVR12 according to basal fibrosis stage. SVR12, sustained virologic response 12 weeks' post-treatment; SOF/VEL \pm RBV, sofosbuvir/ velpatasvir土ribavirin; GLE/PIB, glecaprevir/pibrentasvir.

or the safety of the two treatment regimens. Our results are similar to those observed in the pivotal phase III clinical trials ASTRAL-3 for SOF/VEL \pm RBV $^{27}$ and ENDURANCE-3 for GLE/PIB, ${ }^{29}$ where the overall SVR12 rate was $95 \%$. Although there are no direct comparative observational studies in the routine clinical practice between both fixed combinations of antivirals in G3-HCV-infected patients, some studies have independently evaluated their effectiveness in clinical practice, and most studies found SVR12 results comparable to those found in our study. ${ }^{35-40}$ In addition to the consistency of these results in different populations, it is informative to analyse the virologic response according to the presence of cirrhosis, advanced fibrosis and previous treatment because these factors are used to stratify treatment protocols in international treatment guidelines. ${ }^{17} 18$ In relation to the effectiveness of both drugs in cirrhosis patients in our study and in contrast with other previous observational studies, ${ }^{38} 394142$ we observed a lower SVR12 than in non-cirrhosis patients, although no significant differences were observed between the two drug combinations. This is probably due to the small number of cirrhosis patients included in our study. Therefore, no definitive conclusions can be drawn regarding this subpopulation. However, with respect to cirrhosis patients included in our study, it should be noted that the cutoff point of the FibroScan which we used to categorise patients as cirrhosis was $>14.4 \mathrm{kPa}$ in HCV mono-infected patients and $>14.0 \mathrm{kPa}$ in HIV co-infected patients, whereas in the pivotal clinical studies of SOF/VEL \pm RBV or GLE/PIB it was $12.5 \mathrm{kPa}$. Therefore, patients in our study with advanced F3 fibrosis could have been included in the F4 category according to the criteria used in pivotal clinical trials. In this sense, we observed a significantly lower effectiveness $(\mathrm{P}=0.03)$ of SOF/VEL $\pm \mathrm{RBV}$ and $\mathrm{GLE} /$ PIB in patients with advanced fibrosis F3-4 (86\%), compared with patients with low fibrosis F0-2 (100\%). We observed a high effectiveness of both treatments in treatment-naïve and treatment-experienced patients, without statistically significant differences between SOF/VEL \pm RBV vs GLE/PIB, although it should be considered that only six patients included in this study had received previous antiviral treatment and that only one of them had cirrhosis.

In relation to safety, it is noteworthy that no patient experienced SAEs, visited the Emergency Department, was hospitalised or discontinued antiviral treatment, secondary to SAEs, so we consider that SOF/VEL \pm RBV and GLE/PIB are safe in G3-HCVinfected patients treated in real clinical practice. No significant clinical differences in safety were observed when the two treatment regimens were compared. The rates of any grade of AEs and moderate AEs was lower than those observed in pivotal clinical trials, for both SOF/VEL \pm RBV and GLE/PIB, although the types of AEs reported were similar. In the case of $S O F / V E L \pm R B V$, any degree of AEs observed in clinical trials was $50 \%,{ }^{27}$ whereas in our study it was about half of this figure (26\%), and in the rate of moderate-to-severe AEs was also considerably lower in our study. In relation to GLE/PIB, the rates and the nature and degree of any AEs observed in the patients of our study (13\%) were similar to those observed in the pivotal clinical trials $(8 \%-11 \%) .^{29}$

There was a high adherence to antiviral treatment observed in our study, as has been documented in previous studies, ${ }^{43}$ without differences by drug regimen, and therefore the effectiveness and safety shown by our results were not biased by patients not receiving the intervention. However, surprisingly, a higher incidence of DDIs was observed with SOF/VEL \pm RBV than with GLE/PIB. The latter could be expected to be associated with a higher incidence of DDIs because it contains a protease inhibitor (GLE) that usually has a higher rate of interactions. ${ }^{44}$ We consider that there may have been a selection bias in favour of $\mathrm{SOF} / \mathrm{VEL} \pm \mathrm{RBV}$ in the presence of potential interactions between the basal pharmacological treatment and GLE/PIB, but that this did not have a significant clinical effect because the main interaction was a weak interaction with omeprazole.

This study has the inherent limitations of its design, and it was not possible to perform a multivariate analysis of predictors of response due to the high effectiveness of the treatment and the limited number of study participants. Also, the limited size of the two treatment groups did not allow us to demonstrate clinically 
significant differences regarding the safety of the two treatments, considering that one included ribavirin (SOF/VEL). Of the HCV genotypes, genotype 3 had a lower prevalence in our health area (around 15\%), than that observed in Europe as a who (25\%). ${ }^{2}$ In addition, most G3-HCV patients, especially cirrhosis patients, have already been previously treated and virologically cured using sofosbuvir+ribavirin or sofosbuvir+daclatasvir. These factors limited the size of our study population. Another limitation is the unavailability of data on the Y93 mutation for treatment selection, length of treatment or RBV addition: genotypic resistance testing is not a standard practice in our country before using DAAs to treat cirrhosis. However, currently IDSA/AASLD Recommendations for Testing, Managing, and Treating Hepatitis $\mathrm{C}^{18}$ indicates that NS5A RAS testing is recommended for treatment-naïve patients with cirrhosis infected with g3-HCV, and treatment-experienced patients (with or without cirrhosis) being considered for 12 weeks of sofosbuvir/velpatasvir. Furthermore, if $\mathrm{Y} 93 \mathrm{H}$ is present, weight-based ribavirin should be added or sofosbuvir/velpatasvir/voxilaprevir should be used. However, this study has the strength of being the first comparative study in routine clinical practice. ${ }^{45}$

SOF/VEL \pm RBV and GLE/PIB are both highly effective and safe drug combinations for treating patients with G3-HCV infection, with no significant differences between the two antiviral combinations. Antiviral treatment is less effective in patients with advanced fibrosis.

\section{What this paper adds}

\section{What is already known on this subject}

- Hepatitis C virus genotype 3 is the second most prevalent worldwide and is characterised by developing a hepatic disease steeped in all its phases in relation to infection by other genotypes.

- There is no general consensus on the part of the scientific societies regarding the treatment of choice in these patients, especially in cirrhosis patients or those not responding to previous treatments.

- Sofosbuvir/velpatasvir_ribavirin or glecaprevir/pibrentasbir are the most prescribed treatment in individuals with genotype 3 hepatitis C virus infection.

\section{What this study adds}

- Sofosbuvir/velpatasvir_ribavirin or glecaprivir/pibrentasbir present a high antiviral effectiveness in genotype 3 hepatitis $C$ virus infected patients in real clinical practice, similar than observed in pivotal studies.

- Both direct-acting antivirals show similar effectiveness in these patients, with no significant clinical differences between both antiviral combinations.

- Both antiviral combinations show lower effectiveness in patients with advanced fibrosis F3-4.

- The incidence and profile of adverse events does not differ between sofosbuvir/velpatasvir \pm ribavirin and glecaprevir/ pibrentasvir.

Contributors All authors have contributed to the planning, development and evaluation of the results of this study, as well as their final writing.

Funding The authors have not declared a specific grant for this research from any funding agency in the public, commercial or not-for-profit sectors.

Competing interests LM-F: Honoraria for speaking at symposia. Gilead Sciences, Inc. Janssen. Abbvie Inc. Merck Sharp \& Dohme. Financial support for attending symposia. Gilead Sciences, Inc. Janssen. Abbvie Inc. Merck Sharp \& Dohme. Position on advisory board. Bristol Myers-Squibb. MD-B: Honoraria for speaking at symposia.
Gilead Sciences, Inc. Abbvie Inc. Merck Sharp \& Dohme. Bristol Myers-Squibb. IM-H: Honoraria for speaking at symposia. Gilead Sciences, Inc. Janssen. Financial support for attending symposia. Janssen. Financial support for educational programmes. Janssen. ÁC-I: Honoraria for speaking at symposia. Gilead Sciences, Inc. Abbvie Inc. Financial support for attending symposia. Gilead Sciences, Inc. Abbvie Inc. Merck Sharp \& Dohme. Position on advisory board. Gilead Sciences, Inc.

\section{Patient consent for publication Not required.}

Provenance and peer review Not commissioned; externally peer reviewed.

Data availability statement Data are available upon reasonable request. Data are included in SiMON, an intelligent monitoring system for HCV patients on treatment at A Coruña Hospital.

\section{REFERENCES}

1 European Centre for Disease Prevention and Control. Hepatitis C-Annual epidemiological report for 2015, 2017. Available: https://ecdc.europa.eu/en/ publications-data/hepatitis-c-annual-epidemiological-report-2015 [Accessed 21 Sep 2019].

2 Messina JP, Humphreys I, Flaxman A, et al. Global distribution and prevalence of hepatitis C virus genotypes. Hepatology 2015:61:77-87.

3 Larsen C, Bousquet V, Delarocque-Astagneau E, et al. Hepatitis C virus genotype 3 and the risk of severe liver disease in a large population of drug users in France. $J$ Med Virol 2010;82:1647-54

4 Bochud P-Y, Cai T, Overbeck K, et al. Genotype 3 is associated with accelerated fibrosis progression in chronic hepatitis C. J Hepatol 2009;51:655-66.

5 Adinolfi LE, Gambardella M, Andreana A, et al. Steatosis accelerates the progression of liver damage of chronic hepatitis C patients and correlates with specific HCV genotype and visceral obesity. Hepatology 2001;33:1358-64.

6 De Nicola S, Aghemo A, Rumi MG, et al. HCV genotype 3: an independent predictor of fibrosis progression in chronic hepatitis C. J Hepatol 2009;51:964-6.

7 Nkontchou G, Ziol M, Aout M, et al. HCV genotype 3 is associated with a highe hepatocellular carcinoma incidence in patients with ongoing viral C cirrhosis. J Viral Hepat 2011;18:e516-22.

8 Fartoux L, Poujol-Robert A, Guéchot J, et al. Insulin resistance is a cause of steatosis and fibrosis progression in chronic hepatitis C. Gut 2005;54:1003-8.

9 Leandro G, Mangia A, Hui J, et al. Relationship between steatosis, inflammation, and fibrosis in chronic hepatitis C: a meta-analysis of individual patient data. Gastroenterology 2006;130:1636-42.

10 Kumar D, Farrell GC, Fung C. Hepatitis C virus genotype 3 is cytopathic to hepatocytes: reversal of hepatic steatosis after sustained therapeutic response. Hepatology 2002;36:1266-72.

11 Rubbia-Brandt L, Quadri R, Abid K, et al. Hepatocyte steatosis is a cytopathic effect of hepatitis C virus genotype 3. J Hepatol 2000;33:106-15.

12 Jakobsen JC, Nielsen EE, Feinberg J, et al. Direct-acting antivirals for chronic hepatitis C. Cochrane Database Syst Rev 2017;9:CD012143.

13 Calvaruso V, Craxi A, European Association of the Study of the Liver. 2011 European association of the study of the liver hepatitis $C$ virus clinical practice guidelines. Liver Int 2012;32(Suppl 1):2-8

14 European Association for Study of Liver. EASL clinical practice guidelines: management of hepatitis C virus infection. J Hepatol 2014;60:392-420.

15 European Association for Study of Liver. EASL recommendations on treatment of hepatitis C 2015. J Hepatol 2015;63:199-236.

16 European Association for the Study of the Liver. Electronic address: easloffice@ easloffice.eu. EASL recommendations on treatment of hepatitis C 2016. J Hepato 2017:66:153-94.

17 European Association for the Study of the Liver. Electronic address: easloffice@ easloffice.eu, European Association for the Study of the Liver. EASL recommendations on treatment of hepatitis C 2018. J Hepatol 2018:69:461-511.

18 AASLD-IDSA HCV Guidance Panel. Hepatitis C guidance 2018 update: AASLD-IDSA recommendations for testing, managing, and treating hepatitis $C$ virus infection. Clin Infect Dis 2018;67:1477-92.

19 Margusino-Framiñán L, Cid-Silva P, Mena-de-Cea Álvaro, et al. Intelligent monitoring system for antiviral pharmacotherapy in patients with chronic hepatitis C (SiMON-VC) Farm Hosp 2017;41:68-88.

20 Ministerio de Sanidad, Servicios Sociales e Igualdad, Gobierno de España. INFORME de UTILIDAD TERAPÉUTICA. UT/V1/28022012. Criterios Y recomendaciones generales para El tratamiento Con boceprevir Y telaprevir de la hepatitis crónica C (VHC) en pacientes monoinfectados. Available: https://www.mscbs.gob.es/profesionales/ farmacia/pdf/TRATAMIENTO HEPATITIS_CRONICA C.pdf [Accessed 21 Sep 2019].

21 Gonzalez FA, Van den Eynde E, Perez-Hoyos S, et al. Liver stiffness and aspartate aminotransferase levels predict the risk for liver fibrosis progression in hepatitis $C$ virus/HIV-coinfected patients. HIV Med 2015;16:211-8.

22 Hess LM, Raebel MA, Conner DA, et al. Measurement of adherence in pharmacy administrative databases: a proposal for standard definitions and preferred measures. Ann Pharmacother 2006:40:1280-8.

23 University of Liverpool. Hep drug interactions. Available: https://www.hepdruginteractions.org [Accessed 21 Sep 2019] 
24 Up to Date. Lexicomp drug interactions. Available: https://www.uptodate.com/home/ drugs-drug-interaction [Accessed 21 Sep 2019].

25 IBM Micromedex. Drug interactions. Available: https://www.micromedexsolutions. com/micromedex2/4.29.4.1/WebHelp/Tools/Interactions/Drug_Interactions.htm [Accessed 21 Sep 2019].

26 Wyles D, Bräu N, Kottilil S, et al. Sofosbuvir and velpatasvir for the treatment of hepatitis $C$ virus in patients coinfected with human immunodeficiency virus type 1 : an open-label, phase 3 study. Clin Infect Dis 2017;65:6-12.

27 Foster GR, Afdhal N, Roberts SK, et al. Sofosbuvir and velpatasvir for HCV genotype 2 and 3 infection. N Engl J Med 2015;373:2608-17.

28 Rockstroh JK, Lacombe K, Viani RM, et al. Efficacy and safety of glecaprevir/ pibrentasvir in patients coinfected with hepatitis $C$ virus and human immunodeficiency virus type 1: the EXPEDITION-2 study. Clin Infect Dis 2018;67:1010-7.

29 Foster GR, Gane E, Asatryan A, et al. ENDURANCE-3: safety and efficacy of glecaprevir/pibrentasvir compared to sofosbuvir plus daclatasvir in treatment-naïve HCV genotype 3-infected patients without cirrhosis. J Hepatol 2017;66:S33.

30 Wyles D, Poordad F, Wang S, et al. Glecaprevir/pibrentasvir for hepatitis C virus genotype 3 patients with cirrhosis and/or prior treatment experience: a partially randomized phase 3 clinical trial. Hepatology 2018;67:514-23.

31 Jacobson IM, Lawitz E, Gane EJ, et al. Efficacy of 8 weeks of sofosbuvir, velpatasvir, and voxilaprevir in patients with chronic HCV infection: 2 phase 3 randomized trials. Gastroenterology 2017;153:113-22.

32 Foster GR, Agarwal K, Cramp ME, et al. Elbasvir/grazoprevir and sofosbuvir for hepatitis $C$ virus genotype 3 infection with compensated cirrhosis: a randomized trial Hepatology 2018;67:2113-26.

33 European Medicines Agency. Committee for medicinal products for human use Epclusa. Assessment report, 2017. Available: https://www.ema.europa.eu/en/ documents/assessment-report/epclusa-epar-public-assessment-report_en.pdf [Accessed 21 Sep 2019].

34 European Medicines Agency. Committee for medicinal products for human use Mavyret. Assessment report, 2016. Available: https://www.ema.europa.eu/en/ documents/assessment-report/maviret-epar-public-assessment-report_en.pdf [Accessed 21 Sep 2019].
35 Kumada H, Watanabe T, Suzuki F, et al. Efficacy and safety of glecaprevir/pibrentasvir in HCV-infected Japanese patients with prior DAA experience, severe renal impairment, or genotype 3 infection. J Gastroenterol 2018;53:566-75.

36 Kumada H, Watanabe T, Suzuki F, et al. Correction to: efficacy and safety of glecaprevir/pibrentasvir in HCV-infected Japanese patients with prior DAA experience, severe renal impairment, or genotype 3 infection. J Gastroentero/ 2018;53:689.

37 Flamm S, Mutimer D, Asatryan A, et al. Glecaprevir/pibrentasvir in patients with chronic HCV genotype 3 infection: an integrated phase 2/3 analysis. J Viral Hepat 2019;26:337-49

38 D'Ambrosio R, Pasulo L, Puoti M, et al. Real-world effectiveness and safety of glecaprevir/pibrentasvir in 723 patients with chronic hepatitis C. J Hepatol 2019;70:379-87.

39 Belperio PS, Shahoumian TA, Loomis TP, et al. Real-world effectiveness of daclatasvir plus sofosbuvir and velpatasvir/sofosbuvir in hepatitis C genotype 2 and 3. J Hepatol 2019;70:15-23.

40 Tao Y-C, Deng R, Wang M-L, et al. Satisfactory virological response and fibrosis improvement of sofosbuvir-based regimens for Chinese patients with hepatitis $C$ virus genotype 3 infection: results of a real-world cohort study. Virol J 2018;15:150.

41 Asselah T, Bourgeois S, Pianko S, et al. Sofosbuvir/velpatasvir in patients with hepatitis C virus genotypes 1-6 and compensated cirrhosis or advanced fibrosis. Liver Int 2018;38:443-50

42 Mangia A, Losappio R, Cenderello G, et al. Real life rates of sustained virological response (SVR) and predictors of relapse following DAA treatment in genotype 3 (GT3) patients with advanced fibrosis/cirrhosis. PLoS One 2018;13:e0200568.

43 Burton MJ, Voluse AC, Patel AB, et al. Measuring adherence to hepatitis C directacting antiviral medications: using the VAS in an HCV treatment clinic. South Med J 2018;111:45-50

44 Ottman AA, Townsend ML, Hashem MG, et al. Incidence of drug interactions identified by clinical pharmacists in veterans initiating treatment for chronic hepatitis $C$ infection. Ann Pharmacother 2018;52:763-8.

45 Margusino-Framiñán L, Cid-Silva P, Rotea-Salvo S, et al. PIN3 Sofosbuvir/velpatasvir vs glecaprevir/pibrentasvir in genotype 3 hepatitis C virus infected patients. Value Health 2019:22:S640. 